\title{
Treatment of gout combined with Kienböck's disease: A case report
}

\author{
HUI LU ${ }^{1}$, QIANG CHEN ${ }^{2}$, HANG YU ${ }^{3}$ and ZHENFENG LIU ${ }^{4}$ \\ ${ }^{1}$ Department of Hand Surgery, The First Affiliated Hospital, College of Medicine, Zhejiang University, \\ Hangzhou, Zhejiang 310003; ${ }^{2}$ Department of Hand Surgery, Zhejiang Provincial People's Hospital, Hangzhou, \\ Zhejiang 310014; Departments of ${ }^{3}$ Gastrointestinal Surgery and ${ }^{4}$ PET Center, The First Affiliated Hospital, \\ College of Medicine, Zhejiang University, Hangzhou, Zhejiang 310003, P.R. China
}

Received December 21, 2016; Accepted July 7, 2017

DOI: $10.3892 / e t m .2017 .5039$

\begin{abstract}
Cases of Kienböck's disease combined with gout are rare; to the best of our knowledge, the current report is the third such case reported in the literature. The current report presents the case of a 49-year-old male patient with Kienböck's disease combined with gout. The patient had experienced swelling and pain of their right wrist for 1 month. Through a combination of clinical history, physical examination, laboratory results, radiological and intraoperative findings, the patient was diagnosed with stage IIIB-IV Kienböck's disease and gout. The patient recovered well following a synovectomy and medication for gout. The rarity of the present case makes it difficult to examine the potential association between gout and Kienböck's disease. Kienböck's disease combined with gout tends to be ignored and is easily misdiagnosed. However, the surgical procedure selection for Kienböck's disease combined with gout is markedly different from the conventional surgery for Kienböck's disease or gout alone. The current study outlines a successful treatment strategy for gout combined with Kienbock's disease.
\end{abstract}

\section{Introduction}

Gout is a metabolic disease that is characterized by hyperuricemia. It can attack the first metatarsal joint (1), hand and wrist $(2,3)$. The incidence of gout in the western population is $1-2 \%$ (4). The most common symptom of gout is an acute recurrent attack of inflammatory arthritis. The

Correspondence to: Dr Hang Yu, Department of Gastrointestinal Surgery, The First Affiliated Hospital, College of Medicine, Zhejiang University, 79 Qingchun Road, Hangzhou, Zhejiang 310003, P.R. China

E-mail: 21018059@zju.edu.cn

Abbreviations: MRI, magnetic resonance imaging; CT, computed tomography

Key words: gout, Kienböck's disease, wrist pain metatarsal-phalangeal joint is the most affected joint in almost half of patients. The heels, wrists, knees and fingers can also be affected (5). Gout in the wrist can cause crystal deposits in the hyaline cartilage and synovial membrane, which lead to a reduced vascular supply of the lunate and bone destruction. Hyperuricemia is a feature of gout, but some patients with high uric acid levels never develop gout, and $\sim 50 \%$ of gout patients do not have this symptom (6). Medications to reduce serum uric acid levels can be used.

Kienböck's disease is a disorder of the lunate bone in the wrist, which is characterized by avascular necrosis (7). It is a rare disease, and the incidence of has not been reported. The exact etiology of Kienbock's disease is not known. Median wrist pain is one of the key symptoms. The primary method of treatment is surgery, with the aim of restoring blood supply to the lunate (8). Cases of Kienböck's disease combined with gout are rarely observed (9). To the best of our knowledge, this is the third report in the literature $(9,10)$.

\section{Case report}

In May 2014, a 49-year-old male patient presented to the Department of Hand Surgery at The First Affiliated Hospital of Zhejiang University (Hangzhou, China) with swelling and pain in his right wrist for one month (Fig. 1). The pain had been bearable at first and the patient did not take any medication. At 1 week prior to admission, when the pain became severe, the patient had visited a local hospital. The patient was advised to take non-steroidal anti-inflammatory drugs (Celebrex; Pfizer, Inc., New York, NY, USA; $200 \mathrm{mg}$ twice a day) and given a splint fixation, but there was no notable improvement in pain relief. Upon examination at the present hospital, redness, pain and swelling were noted on the dorsal aspect of his right wrist. Palmar flexion was reduced by $60 \%$ and dorsiflexion was reduced by $80 \%$ compared with the unaffected wrist. The grip strength was $25 \%$ of the unaffected side. The painful pressure spot was located over the central proximal row of the carpal bones. The patient reported no history of trauma. Laboratory test results were performed, including a leukocyte count (11,000 cells $/ \mu \mathrm{l}$; normal range, $4,000-10,000$ cells $/ \mu \mathrm{l})$, uric acid measurement (427 $\mu \mathrm{mol} / 1$; normal range, 208-428 $\mu \mathrm{mol} / 1)$, C-reactive protein concentration assay $(64.30 \mathrm{mg} / \mathrm{dl}$; normal 
range, $0-8 \mathrm{mg} / \mathrm{dl}$ ) and a rheumatological blood screen (rheumatoid factor, $<20 \mathrm{U} / \mathrm{ml}$, normal range, 0-20 U/ml; anti-streptolysin $37 \mathrm{IU} / \mathrm{ml}$, normal range, 0-100 IU/ml) (normal result). Radiographs indicated a blurred joint surface in the distal radius, the proximal pole of the lunate and the scaphoid, narrowing of the joint space, sclerosis and collapse of the lunate bone (Fig. 2). Computed tomography (CT) imaging indicated lunate bone sclerosis with subchondral small cysts, a decrease of lunate height and a narrowing in the joint space of the distal radiocarpal joint (Fig. 3). Magnetic resonance imaging (MRI) revealed low signal intensity in the lunate bone on T1-weighted images and small pieces of high signal intensity on T2-weighted images (Fig. 4). In addition, the synovial tissue exhibited obvious thickening on the dorsal radiocarpal joint (Fig. 4).

The patient was initially diagnosed with stage IIIB-IV Kienböck's disease [according to the classification of Lichtman and Degnan (11)] and synovitis (due to hyperplasia of synovium). An open extra-articular synovectomy was performed, and multiple monosodium urate crystal deposits were observed during the surgery (Fig. 5). A synovectomy with arthroscopy was then performed. The articular surface of the lunate, the lunate facet of the distal radius and the articular surface of the capitate were floating. Synovitis and gouty deposits, and localized articular bone loss were also observed in the arthroscopy. The patient was Grade 3 according to the arthoscopic assessment and classification of Kienböck's disease (12). The final diagnosis was Kienböck's disease (stage IIIB-IV) combined with gout. The patient's wrist swelling had decreased markedly 1 week after the synovectomy with arthroscopy. Bearing heavy loads on the wrist was not recommended. The patient received colchicine (KPC Pharmaceuticals, Inc., Kunming, China; $0.5 \mathrm{mg}$ twice a day) for 1 month after the surgery and was then prescribed allopurinol (Zhejiang Hisun Pharmaceutical Co., Ltd., Taizhou, China; $200 \mathrm{mg}$, three times a day) when the pain had completely ceased. A total of 2 years after the surgery, the patient had no recurrence of wrist pain or limitation of motion, and radiographs revealed no degeneration of the wrist (Fig. 6).

Written informed consent was obtained from the patient for publication of the present case report and accompanying images. Ethical approval was provided by the Medical Ethics Committee of The First Affiliated Hospital of Zhejiang University.

\section{Discussion}

Gout in the wrist can lead to scapholunate dissociation (13), carpal tunnel syndrome and tendon entrapment or rupture (14). To the best of our knowledge, only two cases of Kienböck's disease combined with gout have previously been reported. Castagnoli et al (9) reported the case of 42-year-old male laborer with Kienböck's disease and gout; however, the report did not describe the treatment procedures and only provided the imaging findings. Shin et al (10) reported the case of a 54-year-old patient who presented with stage II Kienböck's disease combined with gout. Arthroscopic synovectomy, intra-articular injection of corticosteroid and partial wrist denervation were performed. The wrist was subsequently pain-free with range of motion and there was no further development of the Kienböck's disease. The two patients described

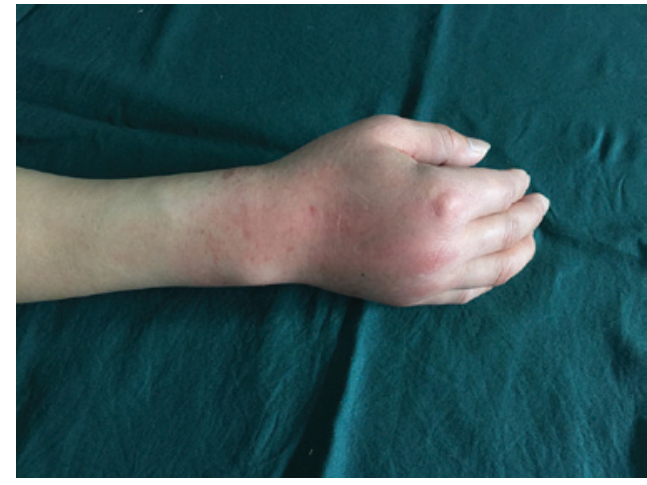

Figure 1. Pre-operative image of the right wrist, which exhibited swelling

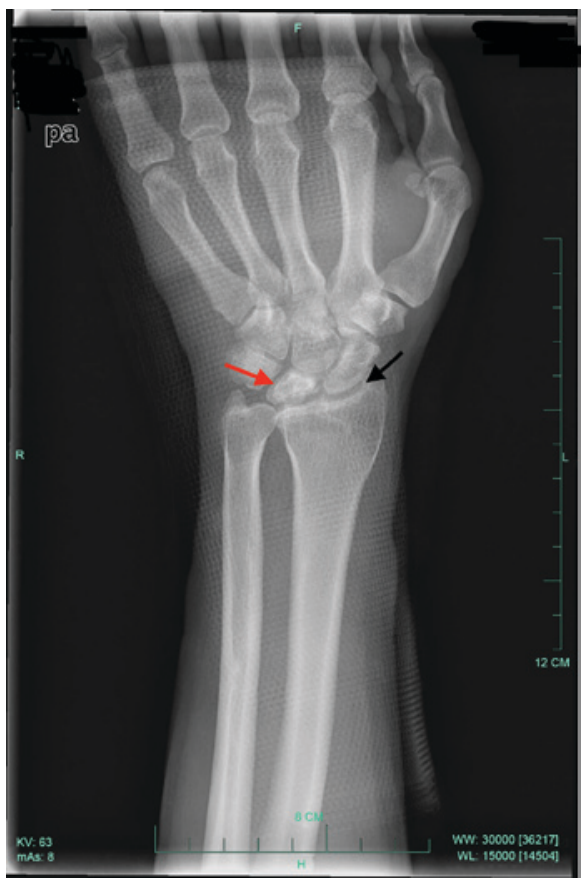

Figure 2. Pre-operative radiograph of the right wrist. Radiographic images revealed a blurred joint surface in the distal radius, the proximal pole of the lunate and the scaphoid (black arrow). In addition, narrowing of the joint space, sclerosis and collapse of the lunate bone were observed (red arrow).

in the aforementioned case reports exhibited hyperuricemia, but the patient in the present study had a normal uric acid level and no history of gout; thus, gout as the initial diagnosis was easily ignored. Monosodium urate crystal deposits observed during the synovectomy made the diagnosis of gout definitive.

The aetiology of Kienböck's disease is unknown, although negative ulnar variance, corticosteroid use, low arterial inflow and trauma are all risk factors for its development (4,15-17). Gout in the wrist can cause crystal deposits in the hyaline cartilage and synovial membrane, which leads to reduced vascular supply of the lunate and bone destruction (10). The concomitant phenomenon of Kienböck's disease and gout may not be a coincidence, and requires further research. Gout may affect blood supply to the lunate, resulting in Kienböck's disease. Resnick et al (18) reported that calcium pyrophosphate dihydrate deposition disease of the lunate bone can appear increasingly opaque compared with normal carpal bone, mimicking the appearance of Kienböck's disease. The diagnosis is characterized 


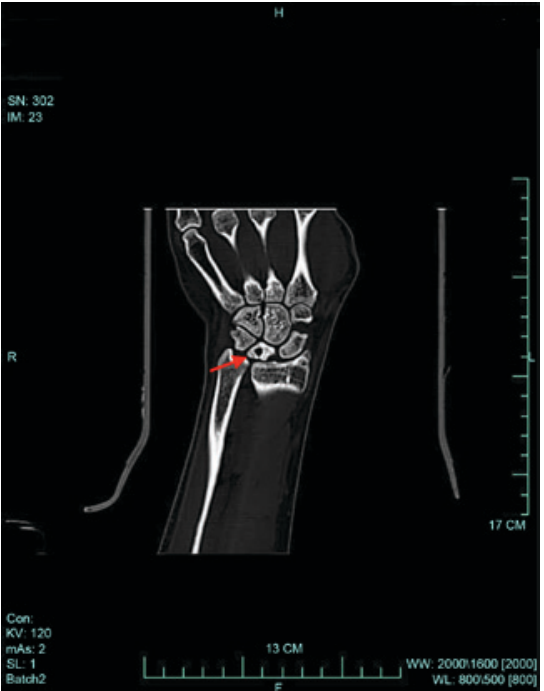

Figure 3. Computed tomography image of the right wrist pre-operatively. Lunate bone sclerosis with subchondral small cysts, a decrease of lunate height and a narrowing joint space of the distal radiocarpal joint were observed (red arrow).

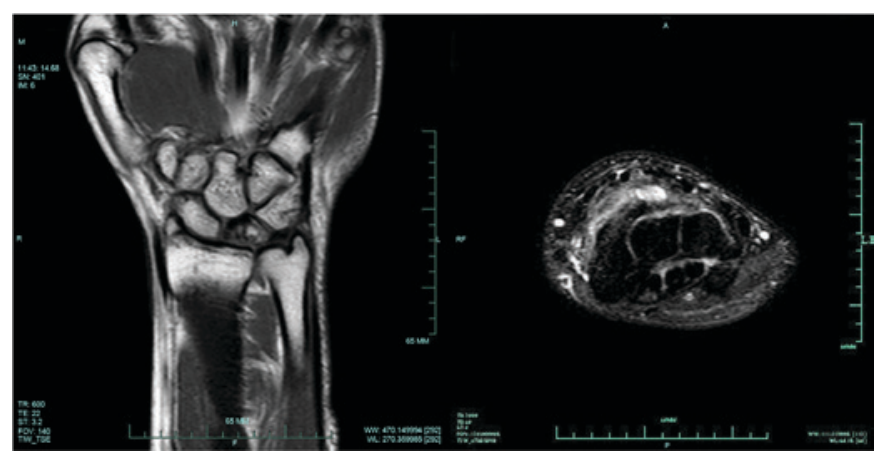

Figure 4. Magnetic resonance imaging of the right wrist pre-operatively. The scan revealed a low signal intensity in the lunate bone on T1-weighted images (left) and small pieces of high signal intensity on T2-weighted images (right). The synovium exhibited obvious thickening on the dorsal radiocarpal joint.

histologically in gout by deposits of monosodium urate crystals. MRI serves a critical role in the diagnosis of Kienböck's disease, and could provide early identification of avascular necrosis of the lunate Marked thickening of the area with synovitis on T2-weighted images in cases of Kienböck's disease combined with gout make it distinct from typical Kienböck's disease.

The primary treatment for Kienböck's disease in patients with gout is to control the symptoms of gout effectively. In the present study, a revascularization procedure or wrist fusion was not performed. Synovectomy for stage II Kienböck's disease (10) and IIIB-IV Kienböck's disease, as in the present case, has been demonstrated to achieve good results. The wrist of the patient in the present study was observed to be more stable compared with ordinary patients with joint degeneration.

In conclusion, the rarity of Kienböck's disease combined with gout makes it difficult to examine the potential association between gout and Kienböck's disease. Kienböck's disease combined with gout tends to be ignored and easily misdiagnosed. Gout may be a potential cause of Kienböck's disease. The surgical procedure chosen for Kienböck's disease

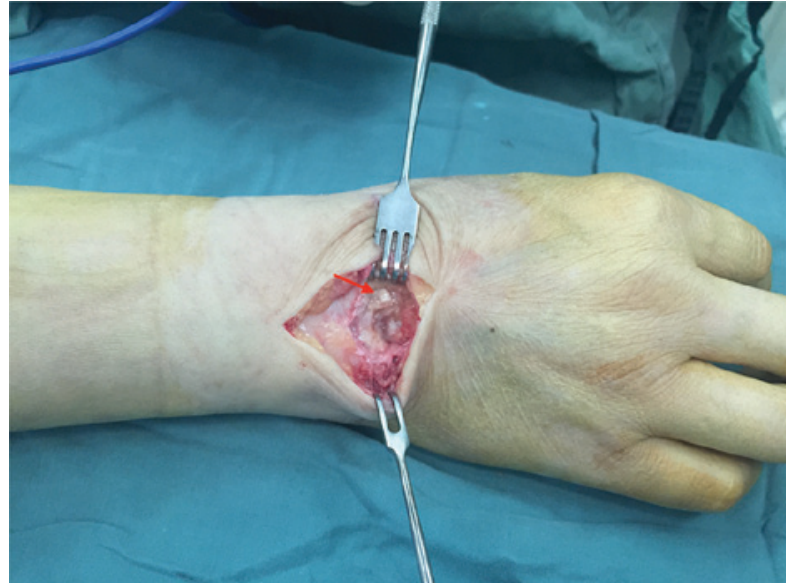

Figure 5. Image of the right wrist during open extra-articular synovectomy, indicating multiple monosodium urate crystal deposits on the synovial membrane of the carpus (red arrow).

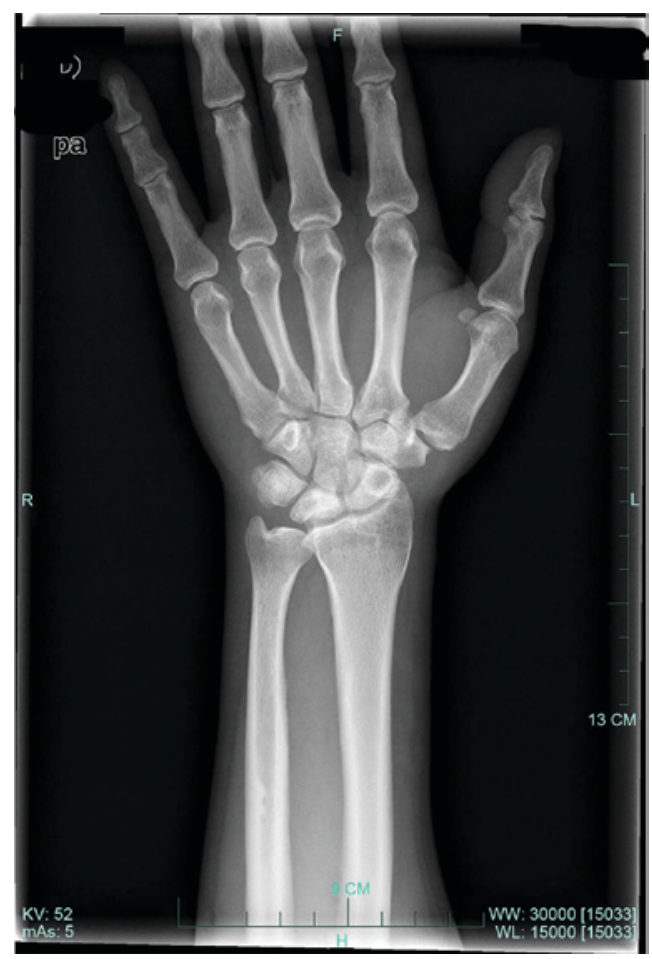

Figure 6. Radiograph performed 2 years after the synovectomy with arthroscopy, revealing no degeneration of the wrist.

combined with gout is markedly different from the conventional surgeries; for instance, more revascularization procedures or wrist fusion are performed for Kienböck's disease alone. The current study outlines a successful treatment strategy for gout combined with Kienbock's disease.

\section{Acknowledgements}

The current study was supported by the Zhejiang Traditional Chinese Medicine Research Program (grant no. 2016ZA124), the Zhejiang Medicine and Hygiene Research Program (grant no. 2016K YB101), the National Natural Science Foundation of China (grant no. 81702135) and the Zhejiang Natural Science Foundation (grant no. LY16H180002). 


\section{References}

1. Hepburn AL and Feher MD: Gout. N Engl J Med 350: 519-520, 2004.

2. Fitzgerald BT, Setty A and Mudgal CS: Gout affecting the hand and wrist. J Am Acad Orthop Surg 15: 625-635, 2007.

3. Kamimura T, Hatakeyama M, Okazaki H and Minota S: Acute gout attack in the wrist joint. Intern Med 43: 641-642, 2004.

4. Beredjiklian PK: Kienbock's disease. J Hand Surg Am 34: 167-175, 2009

5. Schlesinger N: Diagnosing and treating gout: A review to aid primary care physicians. Postgrad Med 122: 157-161, 2010.

6. Sturrock RD: Gout. Easy to misdiagnose. BMJ 320: 132-133, 2000.

7. Dubey PP, Chauhan NK, Siddiqui MS and Verma AK: Study of vascular supply of lunate and consideration applied to Kienböck disease. Hand Surg 16: 9-13, 2011.

8. Tamai S, Yajima $\mathrm{H}$ and Ono $\mathrm{H}$ : Revascularization procedures in the treatment of Kienböck's disease. Hand Clin 9: 455-466, 1993

9. Castagnoli M, Giacomello A, Argentina RS and Zoppini A: Kienböck's disease in gout. Arthritis Rheum 24: 974-975, 1981.

10. Shin AY, Weinstein LP and Bishop AT: Kienböck's disease and gout. J Hand Surg Br 24: 363-365, 1999.
11. Lichtman DM and Degnan GG: Staging and its use in the determination of treatment modalities for Kienböck's disease. Hand Clin 9: 409-416, 1993.

12. Bain GI and Begg M: Arthroscopic assessment and classification of Kienböck's disease. Tech Hand Up Extrem Surg 10: 8-13, 2006.

13. Ohishi T, Koide Y, Takahashi M, Miyata R and Kushida K: Scapholunate dissociation caused by gouty arthritis of the wrist. Case report. Scand J Plast Reconstr Surg Hand Surg 34: 189-191, 2000.

14. Schuind FA, Van Geertruyden J, Stallenberg B, Remmelink M and Pasteels JL: A rare manifestation of gout at the wrist-a case report. Acta Orthop Scand 73: 594-596, 2002.

15. Paksima N and Canedo A: Kienböck's Disease. J Hand Surg Am 34: 1886-1889, 2009.

16. Irisarri C: Aetiology of Kienböck's disease. J Hand Surg Br 29: 281-287, 2004.

17. Watson HK and Guidera PM: Aetiology of Kienböck's disease. J Hand Surg Br 22: 5-7, 1997.

18. Resnick D, Niwayama G, Goergen TG, Utsinger PD, Shapiro RF, Haselwood DH and Wiesner KB: Clinical, radiographic and pathologic abnormalities in calcium pyrophosphate dihydrate deposition disease (CPPD): Pseudogout. Radiology 122: 1-15, 1977. 